\title{
A super lithium-rich red-clump star in the open cluster Trumpler $5^{\star, \star \star}$
}

\author{
L. Monaco ${ }^{1}$, H. M. J. Boffin ${ }^{1}$, P. Bonifacio ${ }^{2}$, S. Villanova ${ }^{3}$, G. Carraro ${ }^{1}$, E. Caffau ${ }^{2,4}$, M. Steffen ${ }^{5,2}$, J. A. Ahumada ${ }^{6}$, \\ Y. Beletsky ${ }^{7}$, and G. Beccari ${ }^{1}$ \\ 1 European Southern Observatory, Casilla 19001 Santiago, Chile \\ e-mail: lmonaco@eso.org \\ 2 GEPI, Observatoire de Paris, CNRS, Univ. Paris Diderot, place Jules Janssen, 92195 Meudon, France \\ 3 Universidad de Concepción, Casilla 160-C, Concepción, Chile \\ 4 Zentrum für Astronomie der Universität Heidelberg, Landessternwarte, Königstuhl 12, 69117 Heidelberg, Germany \\ 5 Leibniz-Institut für Astrophysik Potsdam (AIP), an der Sternwarte 16, 14482 Potsdam, Germany \\ 6 Observatorio Astronómico, Universidad Nacional de Córdoba, Laprida 854, 5000 Córdoba, Argentina \\ ${ }^{7}$ Las Campanas Observatory, Carnegie Institution of Washington, Colina el Pino, Casilla 601, La Serena, Chile \\ Received 28 December 2013 / Accepted 14 March 2014
}

\section{ABSTRACT}

\begin{abstract}
Context. The existence of lithium-rich low-mass red giant stars still represents a challenge for stellar evolution models. Stellar clusters are privileged environments for this kind of investigation.

Aims. To investigate the chemical abundance pattern of the old open cluster Trumpler 5, we observed a sample of four red-clump stars with high-resolution optical spectrographs. One of them (\#3416) reveals extremely strong lithium lines in its spectrum.

Methods. One-dimensional, local thermodynamic equilibrium analysis was performed on the spectra of the observed stars. A 3DNLTE analysis was performed to derive the lithium abundance of star \#3416.

Results. Star \#3416 is super $\mathrm{Li}$-rich with $A(\mathrm{Li})=3.75$ dex. The lack of ${ }^{6} \mathrm{Li}$ enrichment $\left({ }^{6} \mathrm{Li} /{ }^{7} \mathrm{Li}<2 \%\right)$, the low carbon isotopic ratio $\left({ }^{12} \mathrm{C} /{ }^{13} \mathrm{C}=14 \pm 3\right)$, and the lack of evidence for radial velocity variation or enhanced rotational velocity $\left(v \sin i=2.8 \mathrm{~km} \mathrm{~s}{ }^{-1}\right)$ all suggest that lithium production has occurred in this star through the Cameron \& Fowler mechanism.

Conclusions. We identified a super Li-rich core helium-burning, red-clump star in an open cluster. Internal production is the most likely cause of the observed enrichment. Given the expected short duration of a star's Li-rich phase, enrichment is likely to have occurred at the red clump or in the immediately preceding phases, namely during the He-flash at the tip of the red giant branch (RGB) or while ascending the brightest portion of the RGB.
\end{abstract}

Key words. stars: abundances - stars: chemically peculiar - open clusters and associations: individual: Trumpler 5 stars: atmospheres

\section{Introduction}

Lithium is a fragile element that is destroyed at temperatures higher than $2.5 \times 10^{6} \mathrm{~K}$, which may already be reached during the contraction of a protostellar cloud (pre-main sequence phase) that leads to a central hydrogen-burning star. Some depletion is known to take place in the atmosphere of stars similar to the Sun, whose lithium abundance is less than a hundredth the level we observe in meteorites and the interstellar medium (ISM, Population I value). In low-mass stars, additional depletion occurs once the star's atmosphere expands as the star evolves toward the red giant phase. At this stage, the convection in the stellar envelope brings material to the surface from the inner parts. This material was exposed to relatively high temperatures and is, therefore, depleted in lithium.

Observations firmly confirm the scenario depicted above (Gratton et al. 2004), and yet the existence of low-mass stars having lithium abundances in their atmosphere that exceed the prediction of standard stellar models (lithium-rich giants) and even the ISM level (super Li-rich giants) indicates that these stars must synthesize lithium in their interior. Planet engulfment

\footnotetext{
* Based on observations made with ESO Telescopes at the La Silla Paranal Observatory under program ID 088.D-0045(A).

$\star \star$ Appendix $\mathrm{A}$ is available in electronic form at

http://www . aanda.org
}

can be advocated to explain lithium-rich giants, and some suggestive cases have been presented (see, e.g., Adamów et al. 2012a). However, accretion of a planet or a brown dwarf in the stellar envelope would not be able to increase the surface abundance above the Population I value. Additionally, Li-rich giants do not show enhanced ${ }^{9} \mathrm{Be}$ abundances, which would be expected if lithium enrichment is due to planet accretion (Melo et al. 2005). Coupled with adequately modeled mixing in the stellar atmosphere, the Cameron \& Fowler (1971, hereafter CF71) mechanism is considered a viable way to produce fresh lithium in intermediate-mass asymptotic red giants and low-mass red giants. The stellar convective envelope is first enriched in ${ }^{3} \mathrm{He}$ after the first dredge up. The latter can be burned into ${ }^{7} \mathrm{Be}$ if it is transported to regions with high enough temperature, like the hydrogen-burning shell. ${ }^{7} \mathrm{Be}$ then decays into ${ }^{7} \mathrm{Li}$, which should be brought to the stellar surface on a fast enough timescale to escape destruction. Lithium will then be depleted again. The lithium-rich phase would, therefore, be a short-lived one, which would explain the low frequency $(\sim 1 \%)$ of these kinds of stars (de la Reza \& Drake 2012).

The identification of the sites responsible for this production is, however, challenging. Early indications supported the red giant branch luminosity bump for low-mass stars and the asymptotic giant branch clump for intermediate-mass stars, as the preferred evolutionary stages for lithium production 
(Charbonnel \& Balachandran 2000). There are suggestions of possible clustering of Li-rich objects among central He-burning stars (Kumar et al. 2011), while indications that there are lithium-rich stars all along the red giant branch (RGB) sequence have also emerged (Monaco et al. 2011). Most of these studies were, however, limited by the lack of a proper evolutionary status determination.

For these kinds of investigations, stellar clusters are, therefore, privileged environments, since they are single stellar populations, whose stars share a common distance, age, and metallicity. The evolutionary stages of cluster's stars are also readily identified. We present here our identification of a super Li-rich, core-helium-burning red-clump star in the old, massive open cluster Trumpler 5 (Kałużny 1998).

\section{Observations, data reduction, and analysis}

Observations of the open cluster Trumpler 5 were made during the nights of February 11 and 29, 2012 using the multi-object fiber-fed FLAMES facility mounted on the ESO-VLT/UT2 telescope at the Paranal observatory (Chile). Two 2400 s exposures were taken simultaneously for five likely red-clump stars (see Fig. 1) using the UVES high-resolution spectrograph red arm. The spectra cover the 476-684 $\mathrm{nm}$ wavelength range and provide a resolution of $R \simeq 47000$. Data were reduced using the ESO CPL based FLAMES-UVES pipeline ${ }^{1}$. For Star \#3416, additional data were obtained on October 19, 2013 with the MIKE/MAGELLAN high-resolution spectrograph using a $0.7^{\prime \prime}$ slit, corresponding to a resolving power of $R \simeq 42000$. Five $1800 \mathrm{~s}$ exposures were gathered. The spectra were reduced using the MIKE pipeline ${ }^{2}$ and cover the wavelength range 353-940 nm. UVES and MIKE spectra of each star were finally corrected to the rest frame and combined. UVES and MIKE spectra of Star \#3416 were not combined. Radial velocities were computed using the IRAF/fxcor ${ }^{3}$ task to cross correlate the observed spectra with a synthetic one from the Coelho et al. (2005) library with $T_{\text {eff }}=5250 \mathrm{~K}, \log g=2.5$, solar metallicity, and no $\alpha$-enhancement.

The local thermodynamic equilibrium (LTE) line analysis and spectrum synthesis code MOOG (Sneden 1973) was used to perform the abundance analysis adopting 1D ATLAS9 model atmospheres (Kurucz 1993a; Sbordone et al. 2004). Estimates of the atmospheric parameters were derived from the Kałużny (1998, hereafter K98) photometry (see Table A.1). We also adopted their reddening and distance. For the effective temperature $\left(T_{\text {eff }}\right)$ and microturbulence $(\xi)$, we employed the Alonso et al. (1999) and Marino et al. (2008) calibrations, respectively. The stellar surface gravity $(\log g)$ was derived by comparison with theoretical isochrones from the Girardi et al. (2002) collection with a $Z=0.008$ metallicity (Piatti et al. 2004). These initial parameters were then refined following the procedure outlined in Monaco et al. (2011). Elemental abundances were then derived from line equivalent widths or by spectrosynthesis as required, and are reported in Table A.2 (see Monaco et al. 2011; Villanova $\&$ Geisler 2011). Carbon, nitrogen, and oxygen abundances as well as the ${ }^{12} \mathrm{C} /{ }^{13} \mathrm{C}$ carbon isotopic ratio, were determined from the MIKE spectrum for Star \#3416 alone.

\footnotetext{
1 http://www.eso.org/sci/software/pipelines/

2 http://web.mit.edu/ burles/www/MIKE/

3 IRAF is distributed by the National Optical Astronomy Observatories, which are operated by the Association of Universities for Research in Astronomy, Inc., under cooperative agreement with the National Science Foundation.
}

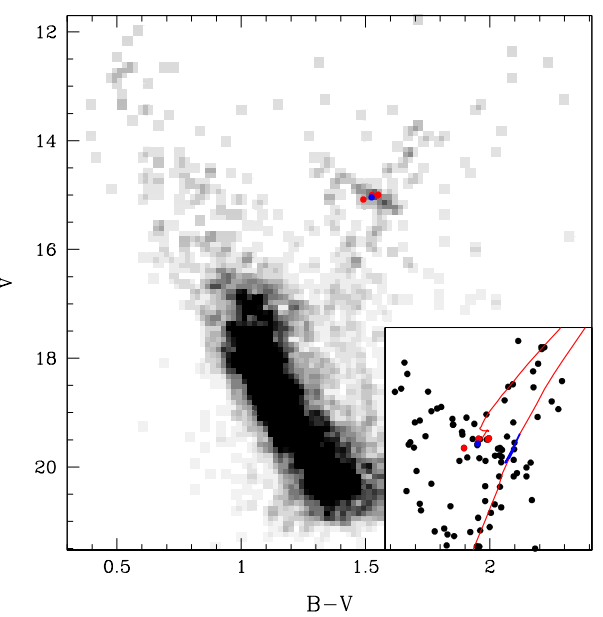

Fig. 1. Trumpler $5 \mathrm{~V}$ vs. $B-V$ color-magnitude diagram from K98. Target stars are marked as colored points. The super Li-rich Star \#3416 is marked in blue. The inset presents a zoomed CMD around the clump region with an isochrone superimposed (Girardi et al. 2002), adopting the K98 parameters and a $Z=0.008$ metallicity. See Sect. 3 for further details.

Second-epoch spectra of Stars \#4649 and \#4791 were of low quality and were, therefore, not combined. For these two stars we analyzed spectra of only the first epoch. Before the analysis, to increase the $\mathrm{S} / \mathrm{N}$, these two stars' spectra were broadened adopting a Gaussian of $F W H M=3.4 \mathrm{~km} \mathrm{~s}^{-1}$. Given the lower quality of their spectra, we adopted as $T_{\text {eff }}$ the initial value for these stars, corrected by $-30 \mathrm{~K}$, which is the mean difference between the initial estimate and the values eventually adopted for the remaining stars. We classify Star \#4649 as non-member of the cluster because its iron content differs from that of the other stars (see Table A.1), and no further analysis was performed on this star.

Lithium abundances or upper limits were derived from synthesis of the resonance doublet at $670.78 \mathrm{~nm}$. For star \#3416, we also measured the subordinate line at $610.36 \mathrm{~nm}$. We calculated the lithium abundances corrections due to NLTE effects, according to Lind et al. (2009, see Table A.1).

To analyze the lithium lines in the Li-rich Star \#3416, we also made use of the 3D hydrodynamical simulations computed with the $\mathrm{CO}^{5}$ BOLD code (Freytag et al. 2012) available in the CIFIST grid (Ludwig et al. 2009). We computed departures from the LTE with the NLTE3D code of Cayrel \& Steffen, as described in Sbordone et al. (2010). The line profiles were computed with the Linfor3D code ${ }^{4}$. In the CIFIST grid there is no simulation with the atmospheric parameters of Star \#3416. We therefore used four bracketing models, with $T_{\text {eff }}=4500 \mathrm{~K} / 5000 \mathrm{~K}$ and $[\mathrm{M} / \mathrm{H}]=0.0 /-1$, and $\log g=2.5$. For each simulation we computed several profiles with different $\mathrm{Li}$ abundances and then fitted the profile to the observed UVES spectrum. In the fitting we also allowed for a Gaussian broadening above the instrumental profile. Although the profiles from the hydrodynamical models already contain a broadening due to macroturbulence, there may be extra broadening due to stellar rotation. We interpret the additional broadening as entirely due to rotation. After deriving the $\mathrm{Li}$ abundance and the broadening for each simulation we did a bilinear interpolation to obtain the abundance and extra broadening for the atmospheric parameters of Star \#3416. The result is $A(\mathrm{Li})=3.75 \mathrm{dex}$ and $v \sin i=2.8 \mathrm{~km} \mathrm{~s}^{-1}$. The best fit for the model with $T_{\text {eff }}=5000 \mathrm{~K}$ and solar metallicity is shown in Fig. 2 (bottom panel). With the broadening obtained from the

4 http://www.aip.de/ mst/linfor3D_main.html 
resonance line we also fit the subordinate $610.3 \mathrm{~nm}$ line and we obtained exactly the same Li abundance (see Fig. 2, top panel).

The 3D-NLTE lithium abundance derived for this star is 0.15 dex higher than the 1D-NLTE value for the resonance $670.7 \mathrm{~nm}$ doublet and 0.09 dex higher for the subordinate Li I $610.3 \mathrm{~nm}$ line. The simplest interpretation of this difference is that the hydrodynamical model correctly takes the microturbulence into account, as shown by the fact that the two lines provide the same abundance. Instead, the $1 \mathrm{D}$ analysis assumes too much microturbulence: a lower assumed microturbulence would result in a higher $\mathrm{Li}$ abundance and a suitable value could force the agreement between the two lines. It should nevertheless be noted that both lines are saturated and located on the flat part of the curve of growth. That a microturbulent value derived from Fe I lines is not suitable for measuring lines of other elements is not surprising and simply shows that the complexity of the velocity fields in a stellar atmosphere cannot be modeled by a single parameter. We further notice that the 1D-LTE synthetic profile does not reproduce the core of the $670.7 \mathrm{~nm}$ line. For any interpetation of the Li abundance in this star, the 3D-NLTE abundance should be used.

We adopt a conservative 0.1 dex as the abundance uncertainty corresponding to the synthetic spectrum fitting. $A \pm 100 \mathrm{~K}$ variation in the model atmosphere $T_{\text {eff }}$ corresponds to a similar abundance change of about \pm 0.1 dex. In Fig. 2 we also show a synthetic spectrum computed with the same total $\mathrm{Li}$ abundance, but $2 \%$ of ${ }^{6} \mathrm{Li}$. The extra depression on the red wing in the latter case is obvious. We can confidently exclude the fractional ratio of ${ }^{6} \mathrm{Li}$ of this star is higher than $2 \%$.

\section{Discussion}

Stellar clusters provide easy access to a wealth of information about the basic parameters of stars in the underlying stellar population (evolutionary stage, age, mass, and metallicity) and are, in principle, ideal environments for investigating the phenomenon of lithium enrichment in giant stars. Unfortunately, specifically devised investigations (Pilachowski et al. 2000) have provided negative results.

A few lithium-rich giants have been, however, identified in clusters, both open and globular (Carney et al. 1998; Hill \& Pasquini 1999; Kraft et al. 1999; Ruchti et al. 2011; Anthony-Twarog et al. 2013). They are generally found along the RGB, apart for the Population II Cepheid V42 discovered by Carney et al. (1998) in the globular cluster M5. Here we have presented the detection of a super-lithium-rich star $(A(\mathrm{Li})=3.75 \mathrm{dex})$, among core He-burning red-clump stars in the open cluster Trumpler 5.

Traditionally, three possible causes of lithium enrichment beyond the level allowed by standard stellar evolution theory models have been suggested: preservation of the original lithium content, pollution by an external source, for instance, the engulfment of a planet or a brown dwarf and, finally, internal production through the CF71 mechanism.

In the present case, the first possibility is excluded by the abundance higher than the ISM level in Star \#3416. Additionally, Trumpler 5 is very similar to the open cluster NGC 2243 in terms of age and metallicity. Unevolved main sequence stars in this cluster have lithium abundances well below the Population I value $(A(\mathrm{Li})=2.70 \pm 0.20$, François et al. 2013), in agreement with the abundance measured at similar metallicity in the Small Magellanic Cloud ISM (Howk et al. 2012). It is, therefore, reasonable to think that Trumpler 5 has a similar original lithium abundance. Furthermore, the low measured ${ }^{12} \mathrm{C} /{ }^{13} \mathrm{C}$ ratio $(14 \pm 3)$ indicates that this star underwent the internal mixing

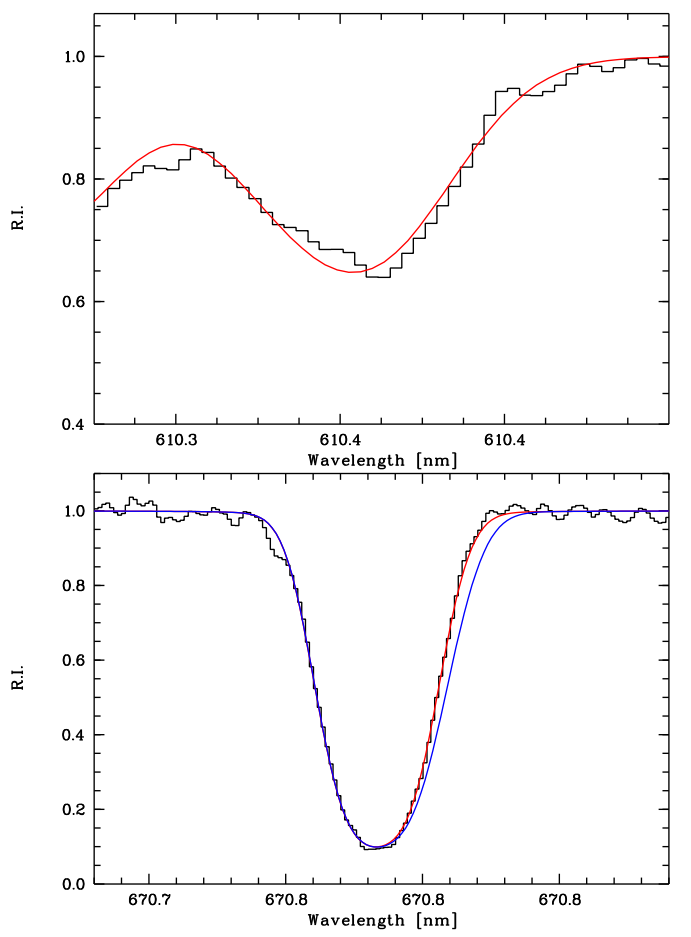

Fig. 2. Sample of the UVES spectrum of Star \#3416 in the region of the Li I resonance doublet (bottom panel) and subordinate line (top panel). Synthetic 3D-NLTE spectra are shown for the same Li abundance in both panels. In the bottom panel also a spectrum computed with $2 \%$ of ${ }^{6} \mathrm{Li}$ is shown.

episodes associated with the first dredge-up and the RGB-bump level (see Gratton et al. 2004). This is common for Li-rich giants, which do present evidence of the mixing they have experienced (Charbonnel \& Balachandran 2000).

In a similar vein, lithium enrichment due to a planet engulfment episode would not be able to raise the star's atmospheric abundance above the ISM value. This should also be recognizable by the presence of a large fraction of ${ }^{6} \mathrm{Li}$, which we rule out for Star \#3416. Increase in ${ }^{6} \mathrm{Li}$ is also foreseen for lithium enrichment due to stellar flares (Tatischeff \& Thibaud 2007), which can also be ruled out as a cause of enrichment. Additionally, Star \#3416 presents a slow rotational velocity $\left(2.8 \mathrm{~km} \mathrm{~s}^{-1}\right)$, and we did not find any indication of binarity - the radial velocities measured on the two UVES epochs and the MIKE observations are compatible with no variation $\left(\Delta v_{\text {helio }}<1 \mathrm{~km} \mathrm{~s}^{-1}\right)$, given the errors in the measurements.

A significant lithium production must then have occurred in the stellar interior of Star \#3416. For the three other stars in Trumpler 5, we measure lithium abundances $A(\mathrm{Li})<1.3 \mathrm{dex}$ (see Table A.1). Star \#3416 thus shows a factor 280 increase in its surface lithium abundance, compared to other red-clump stars in Trumpler 5.

At which evolutionary phase was the lithium produced? Given the expected short lifetime of the Li-rich phase of a star and its high abundance, we may consider that enrichment in \#3416 appeared either during the current red-clump phase or just before, i.e. at the tip of the RGB and its connected core He-flash or on the brightest portion of the RGB. Trumpler 5 redclump stars have, in fact, a current mass range of $\sim 1-1.4 M_{\odot}$ (see Fig. 1), as derived by the comparison with isochrones from the Girardi et al. (2002) collection and adopting the K98 parameters in terms of distance, reddening and age ( $4 \mathrm{Gyr})$. Other cases of lithium-rich giants associated with the red-clump phase have 
been suggested (Ruchti et al. 2011; Kumar et al. 2011; Adamów et al. 2012b; Martell \& Shetrone 2013), but, being field stars, they lacked any firm determination of their evolutionary status.

A preferred association of low-mass lithium-rich giants with the RGB-bump was proposed by Charbonnel \& Balachandran (2000). They argue that removing the mean molecular weight discontinuity left over by the first dredge-up occurring at the RGB-bump led to the onset of the extra mixing between the hydrogen-burning shell (HBS) and the envelope, allowing the CF71 mechanism to become an effective route for producing lithium-rich giants. Indeed, it is now accepted that the onset of thermohaline mixing at the RGB-bump will induce extra mixing between the convective envelope and the stellar interior (see Eggleton et al. 2008; Charbonnel \& Lagarde 2010, and references therein). The more robust sample studied by Kumar et al. (2011, hereafter KRL11), on the other hand, supports an association with the red-clump region and lithium production associated with the He-flash at the RGB tip.

We notice that a number of lithium-rich and super lithiumrich giants have been identified in the brightest portions of the RGB (Kraft et al. 1999; Monaco \& Bonifacio 2008; Monaco et al. 2011; Ruchti et al. 2011; Martell \& Shetrone 2013), therefore the Charbonnel \& Balachandran (2000) or the KRL11 proposals are unable to embrace the whole complex phenomenology of lithium-rich giants, nor do they try to account for it (see Charbonnel \& Balachandran 2000). Nevertheless, the existence of such a preferred association would be an important feature. That extensive surveys for Li-rich giants (Monaco et al. 2011; Ruchti et al. 2011; Martell \& Shetrone 2013) have failed to detect it calls for an explanation. This is, however, beyond the scope of the present paper. Suffice it to say here that the different studies may be covering different parameter spaces. As an example, the bulk of lithium-rich giants in the KRL11 work have $T_{\text {eff }}$ between $\sim 4500$ and $5000 \mathrm{~K}$ and $\log g$ between 1.3 and 2 (see their Fig. 2, bottom panel), which is a region poorly sampled by the Martell \& Shetrone (2013) survey (see their Fig. 1).

It is unclear at present if current stellar models are capable of producing a significant amount of lithium during the He-flash. We remark here that our stars very likely have lower masses than the KRL11 ones, and a lower amount of ${ }^{3} \mathrm{He}$ might remain in the stellar envelope to allow for the CF71 mechanism to operate, after the depletion operated by thermohaline mixing (Eggleton et al. 2008). Therefore, different mechanisms may have operated to produce the lithium abundances observed in KRL11 stars and in Star \#3416 in Trumpler 5.

Indeed, the Denissenkov (2012, hereafter D12) models do not allow for lithium production during the He-flash (see their discussion but also Mocák et al. 2011a,b, for a possible alternative scenario). D12 proposes, alternatively, that lithium-rich stars identified as belonging to the red clump in the KRL11 sample may, in fact, be close to the RGB bump. but that some internal extra-mixing, perhaps related to fast internal rotation, may cause the stars to perform an excursion in the HR diagram towards redder and fainter magnitudes, compatible with the expected location of red-clump stars with masses of $\sim 2 M_{\odot}$.

This scenario may apply to all the lithium-rich giants tentatively identified in the field as red-clump stars. It fails, however, to explain the Trumpler 5 case. Stars compatible with the D12 hypothesis would be found at redder colors than the red clump and at fainter magnitudes, but this location is incompatible with Star \#3416. While the present paper was in the refereeing stage, the detection in the Kepler field of a corehelium-burning lithium-rich giant $\left(A(\mathrm{Li})_{\mathrm{NLTE}}=2.71\right)$ confirmed by asteroseismic analysis was reported (Silva Aguirre et al. 2014). As for Star \#3416, this giant is not carbon rich, and it presents a low carbon isotopic ratio $\left({ }^{12} \mathrm{C} /{ }^{13} \mathrm{C}<20\right)$. These two stars, then provide definitive confirmations of the existence of low-mass lithium-rich and super-lithium-rich stars among redclump stars.

The D12 model may, nevertheless, be at work in other cases. In this respect, it is important to notice the detection of a lithiumrich giant at a level below the RGB-bump in the open cluster NGC 6819. This location may indeed be explained by the D12 model (Anthony-Twarog et al. 2013).

Acknowledgements. P.B. acknowledges support from the CNRS-INSU PNCG. S.V. gratefully acknowledges the support provided by FONDECYT N. 1130721. E.C. is grateful to the MERAC foundation for funding her fellowship. J.A.A. is grateful to ESO for supporting his visit to the Santiago premises in 2010 and 2011, where this project started.

\section{References}

Adamów, M., Niedzielski, A., Villaver, E., Nowak, G., \& Wolszczan, A. 2012a, ApJ, 754, L15

Adamów, M., Niedzielski, A., \& Wolszczan, A. 2012b, Mem. Soc. Astron. It. Suppl., 22, 48

Alonso, A., Arribas, S., \& Martínez-Roger, C. 1999, A\&AS, 140, 261

Anthony-Twarog, B. J., Deliyannis, C. P., Rich, E., \& Twarog, B. A. 2013, ApJ, 767, L19

Cameron, A. G. W., \& Fowler, W. A. 1971, ApJ, 164, 111 (CF71)

Carney, B. W., Fry, A. M., \& Gonzalez, G. 1998, AJ, 116, 2984

Charbonnel, C., \& Balachandran, S. C. 2000, A\&A, 359, 563

Charbonnel, C., \& Lagarde, N. 2010, A\&A, 522, A10

Coelho, P., Barbuy, B., Meléndez, J., Schiavon, R. P., \& Castilho, B. V. 2005, A\&A, 443, 735

de la Reza, R., \& Drake, N. A. 2012, Circumstellar Dynamics at High Resolution, ASP Conf. Ser., 464, 51

Denissenkov, P. A. 2012, ApJ, 753, L3 (D12)

Eggleton, P. P., Dearborn, D. S. P., \& Lattanzio, J. C. 2008, ApJ, 677, 581

François, P., Pasquini, L., Biazzo, K., Bonifacio, P., \& Palsa, R. 2013, A\&A, 552, A136

Freytag, B., Steffen, M., Ludwig, H.-G., et al. 2012, J. Comput. Phys., 231, 919

Girardi, L., Bertelli, G., Bressan, A., et al. 2002, A\&A, 391, 195

Gratton, R., Sneden, C., \& Carretta, E. 2004, ARA\&A, 42, 385

Hill, V., \& Pasquini, L. 1999, A\&A, 348, L21

Howk, J. C., Lehner, N., Fields, B. D., \& Mathews, G. J. 2012, Nature, 489, 121 Kałużny, J. 1998, A\&AS, 133, 25 (K98)

Keeping, E. S. 1962, Introduction to Statistical Inference (Princeton: van Nostrand)

Kraft, R. P., Peterson, R. C., Guhathakurta, P., et al. 1999, ApJ, 518, L53 Kumar, Y. B., Reddy, B. E., \& Lambert, D. L. 2011, ApJ, 730, L12 (KRL11)

Kurucz, R. L. 1993a, CD-ROM 13, 18, http: //kurucz.harvard.edu Lind, K., Asplund, M., \& Barklem, P. S. 2009, A\&A, 503, 541

Ludwig, H.-G., Caffau, E., Steffen, M., et al. 2009, Mem. Soc. Astron. It., 80, 711

Marino, A. F., Villanova, S., Piotto, G., et al. 2008, A\&A, 490, 625

Martell, S. L., \& Shetrone, M. D. 2013, MNRAS, 430, 611

Melo, C. H. F., de Laverny, P., Santos, N. C., et al. 2005, A\&A, 439, 227

Mocák, M., Siess, L., Müller, E. 2011a, A\&A, 533, A53

Mocák, M., Meakin, C. A., Müller, E., \& Siess, L. 2011b, ApJ, 743, 55

Monaco, L., \& Bonifacio, P. 2008, Mem. Soc. Astron. It. Suppl., 79, 524

Monaco, L., Villanova, S., Moni Bidin, C., et al. 2011, A\&A, 529, A90

Piatti, A. E., Clariá, J. J., \& Ahumada, A. V. 2004, MNRAS, 349, 641

Pilachowski, C. A., Sneden, C., Kraft, R. P., Harmer, D., \& Willmarth, D. 2000, AJ, 119, 2895

Ruchti, G. R., Fulbright, J. P., Wyse, R. F. G., et al. 2011, ApJ, 743, 107

Sbordone, L., Bonifacio, P., Castelli, F., \& Kurucz, R. L. 2004, Mem. Soc. Astron. It. Suppl., 5, 93

Sbordone, L., Bonifacio, P., Caffau, E., et al. 2010, A\&A, 522, A26

Silva Aguirre, V., Ruchti, G. R., Hekker, S., et al. 2014, ApJ, 784, L16

Sneden, C. 1973, ApJ, 184, 839

Tatischeff, V., \& Thibaud, J.-P. 2007, A\&A, 469, 265

Villanova, S., \& Geisler, D. 2011, A\&A, 535, A31

Page 5 is available in the electronic edition of the journal at http://www . aanda.org 
L. Monaco et al.: A super Li-rich red-clump star in the open cluster Trumpler 5

\section{Appendix A: Detailed species abundances}

Table A.1 reports program stars' basic parameters: coordinates, magnitudes, atmospheric parameters, radial velocities, and spectral signal-to-noise ratio. The derived iron and lithium abundances are also reported.

Table A. 2 reports the detailed species abundances measured for target stars from 1D-LTE analysis. The adopted solar references are also reported.

Table A.1. Program star coordinates, photometry (from K98), adopted atmospheric parameters, and iron and lithium abundances.

\begin{tabular}{lccccccccccc}
\hline \hline ID & $\alpha(\mathrm{J} 2000)$ & $\delta(\mathrm{J} 2000)$ & $V$ & $B-V$ & $\begin{array}{c}T_{\text {eff }} \\
\mathrm{K}\end{array}$ & $\log g$ & $\begin{array}{c}\xi \\
\mathrm{km} \mathrm{s}^{-1}\end{array}$ & {$[\mathrm{Fe} / \mathrm{H}]$} & $A(\mathrm{Li})$ & $\begin{array}{c}S / N \\
\text { at } 607 \mathrm{~nm}^{2}\end{array}$ & $\begin{array}{c}v_{\text {helio }} \\
\mathrm{km} \mathrm{s}^{-1}\end{array}$ \\
\hline 1318 & $06: 36: 53.5$ & $09: 25: 34.6$ & 15.01 & 1.55 & 4730 & 2.20 & 1.20 & -0.49 & $1.23^{a}$ & 33 & $48.1 \pm 0.3^{e}$ \\
$3416^{c}$ & $06: 36: 40.2$ & $09: 29: 47.8$ & 15.04 & 1.52 & 4850 & 2.20 & 1.28 & -0.51 & $(3.75 / 3.75)^{b}$ & 36 & $49.8 \pm 0.1^{e}$ \\
& & & & & & & & & $(3.60 / 3.66)^{a}$ & 103 & $50.5 \pm 0.2^{f}$ \\
$3416^{d}$ & $06: 36: 40.2$ & $09: 29: 47.8$ & 15.04 & 1.52 & 4870 & 2.05 & 1.33 & -0.53 & - & $47.3 \pm 0.1^{e}$ \\
4649 & $06: 36: 48.0$ & $09: 32: 33.5$ & 15.00 & 1.55 & 4750 & 2.25 & 1.02 & -0.27 & - & 14 & $47.1^{e}$ \\
4791 & $06: 36: 33.1$ & $09: 33: 03.7$ & 15.00 & 1.53 & 4800 & 1.90 & 1.30 & -0.48 & $<1.30^{a}$ & 11 & $50.0 \pm 0.1^{e}$ \\
6223 & $06: 36: 38.6$ & $09: 38: 52.6$ & 15.08 & 1.49 & 4830 & 2.40 & 1.21 & -0.42 & $<1.18^{a}$ & 30 & $50.8 \pm 0.4^{e}$ \\
\hline
\end{tabular}

Notes. The spectra signal-to-noise ratios are also indicated, as well as the measured radial velocities. Lithium abundances are derived from the $670.78 \mathrm{~nm}$ line for all stars but for \#3416, for which the values from both the $670.78 \mathrm{~nm}$ and the $610.36 \mathrm{~nm}$ lines are reported as $\left(A(\mathrm{Li})_{670.78 \mathrm{~nm}} / A(\mathrm{Li})_{610.36 \mathrm{~nm}}\right){ }^{(a)}$ Abundance derived with 1D-LTE analysis plus NLTE corrections calculated according to Lind et al. (2009). Owing to the grid boundaries, NLTE corrections were adopted assuming $\xi=2.0 \mathrm{~km} \mathrm{~s}^{-1}$. ${ }^{(b)}$ Value measured from 3D-NLTE analysis. ${ }^{(c)}$ Analysis based on UVES spectrum. ${ }^{(d)}$ Analysis based on MIKE spectrum. ${ }^{(e)}$ Radial velocity uncertainty calculated as the difference between the two epochs $v_{\text {helio }}$ multiplied by 0.63 (small sample statistics, see Keeping 1962). ${ }^{(f)}$ Formal error on radial velocity determination from cross-correlation analysis.

Table A.2. Measured abundances for the program stars.

\begin{tabular}{|c|c|c|c|c|c|c|}
\hline Element & \#1318 & $\# 3416^{a}$ & $\# 3416^{b}$ & \#4791 & \#6223 & Sun \\
\hline$[\mathrm{Fe} \mathrm{I} / \mathrm{H}]$ & $-0.49(107 / 0.12)$ & $-0.51(95 / 0.11)$ & $-0.53(112 / 0.07)$ & $-0.48(33 / 0.14)$ & $-0.42(68 / 0.08)$ & 7.50 \\
\hline$[\mathrm{Fe} \mathrm{II} / \mathrm{H}]$ & $-0.50(11 / 0.13)$ & $-0.53(10 / 0.17)$ & $-0.54(12 / 0.10)$ & $-0.49(7 / 0.17)$ & $-0.44(9 / 0.09)$ & 7.50 \\
\hline$[\mathrm{C} / \mathrm{Fe}]$ & - & - & $-0.17 /-0.07$ & - & - & 8.49 \\
\hline$[\mathrm{N} / \mathrm{Fe}]$ & - & - & $0.05 / 0.15$ & - & - & 7.95 \\
\hline [O/Fe] & - & - & $-0.07(1 /-)$ & - & - & 8.80 \\
\hline${ }^{12} \mathrm{C} /{ }^{13} \mathrm{C}$ & - & - & $14 \pm 3$ & - & - & \\
\hline$[\mathrm{Na} / \mathrm{Fe}]$ & $0.09(2 / 0.06)$ & $0.12(1 /-)$ & $0.18(2 / 0.01)$ & $0.24(1 /-)$ & $0.16(2 / 0.12)$ & 6.32 \\
\hline$[\mathrm{Mg} / \mathrm{Fe}]$ & $0.17(1 /-)$ & $0.25(1 /-)$ & $0.27(1 /-)$ & $0.08(1 /-)$ & $0.17(1 /-)$ & 7.56 \\
\hline$[\mathrm{Al} / \mathrm{Fe}]$ & $0.26(2 / 0.04)$ & $0.22(1 /-)$ & $0.22(1 /-)$ & $0.35(2 / 0.11)$ & $0.25(1 /-)$ & 6.43 \\
\hline$[\mathrm{Si} / \mathrm{Fe}]$ & $0.03(6 / 0.18)$ & $0.06(5 / 0.12)$ & $0.08(8 / 0.07)$ & - & $0.03(5 / 0.06)$ & 7.61 \\
\hline$[\mathrm{Ca} / \mathrm{Fe}]$ & $0.14(6 / 0.08)$ & $0.16(6 / 0.08)$ & $0.18(6 / 0.10)$ & $-0.06(5 / 0.43)$ & $0.10(7 / 0.14)$ & 6.39 \\
\hline$[\mathrm{Ti} / \mathrm{Fe}]$ & $0.17(8 / 0.11)$ & $0.10(10 / 0.13)$ & $0.12(10 / 0.09)$ & $0.16(4 / 0.25)$ & $0.30(8 / 0.09)$ & 4.94 \\
\hline [Ti II/Fe II] & $0.09(2 / 0.03)$ & $0.18(2 / 0.22)$ & - & - & $0.14(2 / 0.02)$ & 4.94 \\
\hline [V/Fe] & $0.30(1 /-)$ & $0.32(1 /-)$ & - & - & $0.24(1 /-)$ & 4.00 \\
\hline$[\mathrm{Cr} / \mathrm{Fe}]$ & $0.00(6 / 0.15)$ & 0.07 (6/0.17) & $0.16(6 / 0.09)$ & - & $0.30(7 / 0.29)$ & 5.63 \\
\hline$[\mathrm{Ni} / \mathrm{Fe}]$ & $0.05(15 / 0.17)$ & $0.02(16 / 0.17)$ & $0(24 / 0.09)$ & $0.05(6 / 0.19)$ & $0.14(18 / 0.19)$ & 6.26 \\
\hline$[\mathrm{Cu} / \mathrm{Fe}]$ & $0.21(1 /-)$ & $0.31(1 /-)$ & $0.25(1 /-)$ & - & - & 4.19 \\
\hline$[\mathrm{Zn} / \mathrm{Fe}]$ & $0.01(1 /-)$ & - & - & - & $-0.18(1 /-)$ & 6.61 \\
\hline [Y II/Fe II] & $-0.07(3 / 0.03)$ & $0.04(3 / 0.08)$ & $0(2 / 0.00)$ & - & $-0.01(3 / 0.33)$ & 2.25 \\
\hline [Zr II/Fe II] & $0.09(1 /-)$ & $0.08(1 /-)$ & $0.05(1 /-)$ & - & $0.04(1 /-)$ & 2.56 \\
\hline [Ba II/Fe II] & $0.06(1 /-)$ & $0.08(1 /-)$ & $0.19(1 /-)$ & $0.17(1 /-)$ & $0.16(1 /-)$ & 2.34 \\
\hline [La II/Fe II] & $0.09(1 /-)$ & $0.19(1 /-)$ & - & - & $-0.10(1 /-)$ & 1.26 \\
\hline [Eu II/Fe II] & $0.27(1 /-)$ & $0.21(1 /-)$ & $0.18(1 /-)$ & $0.19(1 /-)$ & $0.07(1 /-)$ & 0.52 \\
\hline
\end{tabular}

Notes. Adopted absolute $(A(\mathrm{X}))$ solar values are also reported. Numbers in parenthesis indicate the number of lines from which the abundance is derived and the abundance Gaussian $\sigma$ value, when the abundance is derived from more than one line. The two values for the carbon and nitrogen abundances for Star \#3416 are derived from the $G$-band and $\mathrm{C} 2$ band at $563.5 \mathrm{~nm}$ and from the $\mathrm{CN}$ lines at $634 \mathrm{~nm}$ and $800 \mathrm{~nm} .{ }^{12} \mathrm{C} /{ }^{13} \mathrm{C}$ isotopic ratio was measured from $\mathrm{CN}$ lines at $800 \mathrm{~nm} .{ }^{(a)}$ Analysis based on UVES spectrum. ${ }^{(b)}$ Analysis based on MIKE spectrum. 